\title{
Factores de riesgo asociados con alteraciones respiratorias clínicas, espirométricas y radiográficas a seis meses de seguimiento en pacientes con nueva influenza $\mathrm{A}(\mathrm{H} 1 \mathrm{~N} 1)$
}

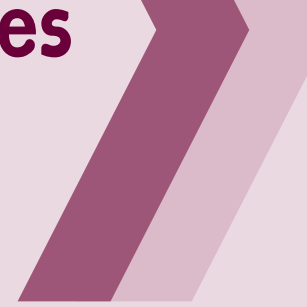

\author{
Risk factors associated to \\ clinical respiratory disorder, \\ spirometric and $x$-ray \\ abnormalities, at six months \\ follow-up in patients with novel \\ influenza A(H1N1)
}

\section{Pablo Grajeda-Ancca, ${ }^{1,4}$ Ronald Sánchez-Lezama, ${ }^{3}$ Kely Quirita-Gonzales,, Oscar F. Niño de Guzmán,,1,2 Manuel Montoya-Lizárraga ${ }^{1,3}$}

\author{
1. Universidad Nacional San Antonio Abad. Cusco, Perú. \\ 2. Hospital Antonio Lorena, Cusco, Perú. \\ 3. Hospital Regional, Cusco, Perú. \\ 4. Field Epidemiology Training Program (FETP), Perú.
}

\section{RESUMEN}

INTRODUCCIón. En 2009, en la Región Cusco se reportaron 395 casos confirmados de nueva influenza A(H1N1) -NIAH1N1-, 15 defunciones, con alta tasa de letalidad (3,8\%). Los efectos posteriores al cuadro agudo son los temas menos investigados, aunque es importante estudiar la presencia de disfunción respiratoria a seis meses de seguimiento. OвJetivo. Determinar los factores de riesgo asociados (FRA) a la presencia y características de las alteraciones respiratorias clínicas, espirométricas y radiográficas al sexto mes de seguimiento en sujetos con antecedente de infección por la NIAHIN1. Métodos. Estudio de corte transversal, en 118 pacientes mayores de 14 años, seis meses después de haber tenido un cuadro de NIAH1N1, confirmado por PCRrt, sin antecedente de afección pulmonar previa en 2009. Resultados. Casi 7 de cada 10 sujetos presentó alguna alteración, las espirométrica y clínica fueron las más frecuentes. La patología de riesgo asociada $(R P=4,11$ [IC95\% $=2,49-6,78], p=0,0000002$ ), la demora en el inicio del tratamiento antiviral específico $(R P=4,56[$ [IC95\% $=2,48$ $8,37], \quad p=0,0000001$ ) y la presencia de insuficiencia respiratoria aguda grave (IRAG) $(R P=11,35$ [IC95\% $=4,27$ 30,17], $p=0,0000000$ ) son FRA a alteraciones clínicas; la patología de riesgo asociada $(R P=12,52$ [IC95\% $=2,7-58,08]$, $p=0,00003)$, un FRA a alteración radiográfica; la presencia de IRAG $(R P=1,33$ [IC95\%=1,02-1,74], $p=0,04)$, un FRA a alteración espirométrica. ConcLusiones. La NIAHIN1 produce alteraciones clínicas, espirométricas y radiográficas a seis meses de presentado el cuadro agudo, y los FRA son patología de riesgo asociada, demora en el inicio del tratamiento antiviral específico y presencia de IRAG, que deben ser considerados para el tratamiento apropiado, y realizar el seguimiento respectivo y prevenir estas alteraciones.

Palabras clave: virus de la influenza A; subtipo H1N1; riesgo.

\begin{abstract}
BACKGRound. In 2009, in Cusco region was reported 395 cases of Novel Influenza $A(H 1 N 1), 15$ deaths (lethality rate $=3,8 \%$ ), becoming serious public health problem. The post-acute effects are least investigated topics, and still scarce literature; to be important, to study the presence of respiratory dysfunction at six months follow-Uр. ОВנEстIVE. To determine the risk factors associated (RFA) to clinical respiratory disorder, spirometric and $x$-ray abnormalities, observed after sixth month follow-up in patients with influenza AH1N1. Meтноos. A cross sectional study was developed in 118 patients over 14 years old, six months after having influenza AH1N1 confirmed by PCRrt test, no history of lung disease before 2009. Results. Nearly 7 of every 10 of patients had some type of modifications, the spirometric and clinical disorders were most frequent. The disease of risk associated $(P R=4,11[95 \% \mid C=2,49-6,78]$, $p=0,0000002$ ), the delay in specific antiviral treatment $(P R=4,56 \quad[95 \% I C=2,48-8,37], P=0,0000001)$, and the presence of severe acute respiratory infection (SARI) ( $P R=11,35$ [95\%IC $=4,27-30,17], p=0,0000000)$ were the RFA to clinical respiratory disorder. The disease of risk associated ( $P R=12,52[95 \% \mid C=2,7-58,08], p=0,00003$ ) was associated to radiographic abnormalities. The presence of SARI (PR $=1,33 \quad[95 \% \mid C=1,02-1,74], \quad p=0,04)$ was associated to spirometric abnormalities. Conclusions. The influenza AH1N1 produces clinical respiratory, spirometric and radiographic abnormalities; which are present six months after the acute episode. There are RFA how the disease of risk associated, delay in the specific treatment, and presence of SARI, that require consideration, to give appropriate treatment and the respective follow, and prevent these abnormalities.
\end{abstract}

KEr words: influenza A virus; H1N1 subtype; risk. 


\section{INTRODUCCIÓN}

La influenza estacional es una de las enfermedades infecciosas que produce cada año una elevada carga de enfermedad, en virtud de las epidemias que ocasiona en todo el mundo. A fines del siglo pasado, en 1997, se empezaron a presentar en países del sureste asiático casos de influenza humana producidos por el virus de la influenza $\mathrm{A}$ (H5N1) de origen aviar, con una letalidad muy alta. Desde entonces, la amenaza de una pandemia de influenza producida por ese virus ha estado latente, a pesar de que hasta ahora, se ha reportado su baja capacidad de transmisión de persona a persona., ${ }^{1,2}$

Entre marzo y abril de 2009, se presentaron casos de síndrome respiratorio agudo grave asociadas a gripe extraña descritos en México y Estados Unidos, las que luego dieron lugar a la identificación de un nuevo subtipo del virus influenza $A$, el que se transmitía de persona a persona, en forma muy similar a como se propagan los virus de la influenza estacional; la que se diseminó rápidamente en el orbe. $^{3}$

El jueves 23 de abril de 2009, la secretaria de Salud del Gobierno de México comunicó al mundo, la aparición de una nueva enfermedad, a la que inicialmente se le denominó como 'gripe o influenza porcina', y luego se le dio la denominación oficial de nueva influenza $A(H 1 N 1)$ [NIAH1N1]. El día 24 de abril, la Dirección General de Epidemiología del Ministerio de Salud del Perú emitió la alerta epidemiológica, lo que generó que se diera inmediatamente la alerta epidemiológica en el ámbito de la Dirección Regional de Salud del Cusco.

En respuesta a los casos de la NIAH1N1 reportados en México, la directora general de la Organización Mundial de la Salud (OMS), Margaret Chan convocó a una reunión del Comité de Emergencias de la OMS, para que evaluara la situación, el cual se estableció de conformidad con lo dispuesto en el Reglamento Sanitario Internacional del 2005. La primera reunión se celebró el sábado 25 de abril de 2009, donde la OMS declaró a la epidemia como una 'emergencia de salud pública internacional'; y el 27 de abril de 2009, elevó el nivel de alerta pandémica a fase 4 , que indica transmisión sostenida de un nuevo virus de influenza animal
- animal-humana de persona a persona que pueda producir 'brotes a nivel de la comunidad de manera sostenida'.4-6 El 29 de abril del 2009, la OMS elevó a fase 5 el nivel de alerta ante una posible pandemia, a la que denomino 'periodo de alerta pandémica'. El organismo hizo un llamado a todos los países a estar preparados para poner en práctica sus planes de contingencia. La fase 5 se establece como: "Uno o varios conglomerados más grandes, con transmisión de persona a persona aún localizada, lo cual indica que el virus se adapta cada vez mejor a los seres humanos, produciendo brotes comunitarios sostenidos en dos o más países de una región de la OMS, pero tal vez no es todavía plenamente transmisible (riesgo pandémico considerable)" .

El 2 de junio de 2009, Keiji Fukuda, asesor de la directora de la OMS, hizo por primera vez, una modificación en la manera de calificar a la enfermedad, dejó de hablar de una influenza de 'gravedad leve', y pasó a calificarla de 'gravedad moderada'. La causa es que, los datos que se disponían "no reflejaban el estado real de la infección". Además, hay características que la hacen especial, se está propagando por el Norte, con la llegada del calor; afecta más a menores de 60 años, los enfermos más graves (los fallecidos o los que desarrollan neumonías que necesitan incluso el uso de respiradores) tienen mayoritariamente entre 20 y 40 años, y hay una parte importante de los fallecidos que no pertenecen a grupos de riesgo (ni son mayores, ni tenían enfermedades crónicas previas, ni eran mujeres embarazadas). ${ }^{8}$

El 11 de junio del 2009, se celebro la cuarta reunión del comité de emergencias, la cual con la información disponible, sobre la transmisión de la NIAH1N1 en cierto número de localidades de países de diferentes regiones de la OMS, llegó a la conclusión de que se cumplían los criterios que definen a una pandemia. La directora general de la OMS decidió elevar el nivel de alerta de pandemia de influenza de la fase 5 a la fase 6 , reservada para la situación más grave, la de una expansión mundial. ${ }^{8}$ La fase 6 (fase pandémica), se caracteriza por los criterios que definen la fase 5 , además de brotes a escala de la comunidad en al menos otro país de una región diferente de la OMS. La declaración de esta fase indica que está 
en marcha una pandemia mundial,, 10 que fue considerada 'incontrolable'.11

Posteriormente se determinó que, el primer caso de la nueva influenza $\mathrm{A}$ ( $\mathrm{H} 1 \mathrm{~N} 1)$ habría sido detectado el 18 de marzo de 2009 en México, extendiéndose en un mes por varios estados de México (Distrito Federal, Estado de México y San Luis Potosí) y luego a EE UU (Texas y California), dos de estos casos iniciales de gripe inusual fueron reportados en EE UU el 18 de abril. Luego surgieron numerosos casos en otros países de pacientes que habían viajado a México, lo demás es historia que ya se conoce. ${ }^{9}$

La NIAH1N1 es una enfermedad respiratoria aguda altamente contagiosa, que apareció por primera vez en México, en marzo de 2009; es causada por un nuevo subtipo del virus de la influenza, que no había circulado nunca en la especie humana y que no tiene ninguna relación con otros virus gripales estacionales, que han afectado anteriormente 0 están afectando al hombre. Este nuevo virus, en un comienzo fue mal llamado como virus de la "gripe o influenza porcina" porque las pruebas de diagnóstico en laboratorios indicaron que muchos de los genes presentes en este virus eran muy similares a los virus de la influenza que afectan con regularidad a los cerdos de América del Norte. Sin embargo, estudios adicionales han indicado que este virus es muy diferente a los que comúnmente circulan entre este tipo de cerdos. Tiene dos genes de cepas de virus de la influenza que circulan comúnmente en cerdos en Europa y Asia, así como un gen de cepa aviar y un gen de cepa humana transmisible al hombre, por lo que los científicos lo denominaron 'virus reordenado cuádruple'.12

A nivel mundial, hasta el 4 de enero de 2010, se habían notificado oficialmente 13290 casos de fallecimiento por infección con el virus de la pandemia de la NIAH1N1, en 137 de los 192 países afectados por la pandemia. Desde el 20 de julio de 2009, ya no se cuentan con un número total de casos, solo se reportan los casos estudiados y comprobados, que son los graves. ${ }^{2,13}$

En América, hasta el 1 de enero de 2010, se han reportado más de 190765 casos y se notificaron un total de 6880 defunciones entre los casos confirmados en 28 países de la región. 14,15
Así mismo, en Perú, hasta el 1 de enero de 2010, se reportaron 9284 casos confirmados y 208 defunciones por la NIAH1N1, con una letalidad de $2,24 \%$. Los casos estuvieron distribuidos en todos los departamentos del Perú, el mayor número ha sido reportado en Lima con 3506 casos, seguido por Arequipa con 1052 casos. Cusco reportó un total de 480 casos confirmados y 15 defunciones, con una alta tasa de letalidad de $3,8 \%$, constituyéndose en serio problema de salud pública emergente. ${ }^{15}$ En Perú, el primer caso se reportó en la ciudad de Lima, el 14 de mayo del 2009:16 en tanto que en Cusco, oficialmente el primer caso se reportó el 14 de junio de 2009.

Además, dado que la NIAH1N1 es una enfermedad emergente sobre la cual hay que generar conocimiento sobre sus características, su evolución y particularmente sobre los factores de riesgo que estén asociados a la mortalidad; porque hay que reconocer que la infección por el virus de la NIAH1N1 puede complicarse y dar lugar a una infección respiratoria aguda grave (IRAG), síndrome de distrés respiratorio agudo (SDRA), que pueden ocasionar una elevada morbimortalidad caracterizada por un inicio agudo, hipoxemia e infiltrados pulmonares bilaterales. El SDRA es la respuesta del pulmón a una agresión local o sistémica, en la que se genera una inflamación local y una alteración de la coagulación, que conduce a un edema inflamatorio pulmonar; que requerirán atención intensiva, la que podría exceder la capacidad de las Unidades de Cuidados Intensivos y afectar la atención de otros daños a la salud. ${ }^{17}$

Las descripciones existentes sobre aspectos fisiopatológicos de la nueva enfermedad reportan la presencia de necrosis alveolar en necropsias de sujetos previamente sanos, así como la existencia de hipertensión pulmonar producto del aumento de la resistencia vascular por la fibrosis perialveolocapilar. ${ }^{18}$

La OMS, el 16 de octubre de 2009, remarca hallazgos de lesiones compatibles con el denominado SDRA, daño alveolar masivo, engrosamiento septal, hiperplasia de neumocitos y presencia de membrana hialina eosinofílica, lo que justifica el grave trastorno en la alveolo 
difusión y su consecuencia, la hipoxia. Estas lesiones se asociaron con bronquiolitis obliterante, neumonía viral primaria, infiltración linfocítica y actividad linfoproliferativa. Datos de laboratorio permitieron valorar el pronóstico evolutivo de estos sujetos, a través de hallazgos importantes como, la elevación conjunta en el suero de tres importantes enzimas: lactato deshidrogenasa, creatinfosfocinasa (CPK) y aminotransferasas (ALT, AST). La elevación importante y conjunta de estas tres enzimas representa una alerta evolutiva que ensombrece el pronóstico y puede anunciar un mayor riesgo de mortalidad; o puede significar una vida con restricciones producto de la patología desencadenada, la que sustenta la presencia de secuelas, que para el virus en particular es de importancia en la salud pública mundial y que amerita un manejo pronto de los pacientes, con fines de mejorar la calidad de vida de los comprometidos. ${ }^{19}$

La decisión de desarrollar esta investigación se dio, debido a que personas que meses atrás habían sido catalogados como casos confirmados de NIAH1N1 venían por un cuadro respiratorio de regular intensidad, y se preguntaban si algo tenía que ver el hecho de haber tenido meses atrás el daño en estudio.

Este hecho obligó a realizar una investigación preliminar de 30 pacientes, de los cuales, $60 \%$ eran del sexo femenino y $40 \%$, del sexo masculino, con edad media de 30 años. Al ingreso, $60 \%$ de estos pacientes tuvieron dificultad respiratoria (saturación de oxígeno $85 \%$ o menos); $70 \%$, fiebre de $39^{\circ} \mathrm{C}$ o más; $90 \%$, compromiso de dos cuadrantes pulmonares con infiltrado intersticial; $70 \%$, algún factor de riesgo (embarazo $13,3 \%$, enfermedad cardiovascular $16,7 \%$, enfermedad pulmonar previa $16,7 \%$, entre otros); en ninguno de los casos existió una prueba de espirometría previa. En cuanto al compromiso pulmonar evidenciado por radiografías de tórax, se apreció el compromiso de dos cuadrantes pulmonares cuando menos, con diferente tipo de infiltrado intersticial, sin que tenga una correlación establecida con el grado de severidad clínica. A la fecha de alta hospitalaria, $80 \%$ de los pacientes tuvieron una saturación de oxígeno menores de $89 \%$. En controles ambulatorios posteriores, el motivo de consulta fue la presencia de disnea a moderado esfuerzo, además, de mantener los valores de saturación de oxigeno menores a $89 \%$.

Sin embargo, llamaba la atención algunos casos particulares. Los pacientes con NIAH1N1 que en su momento habían sido hospitalizados por IRAG con saturación menor de $75 \%$ (e incluso menor de $55 \%$ ), a esa fecha se encontraban asintomáticos con saturaciones de $90 \%$ (3 casos). Pacientes, que habían tenido saturación por encima de $82 \%$, presentaban disnea a moderado esfuerzo ( 5 casos); todo esto considerando que previo a la infección por NIAH1N1 eran personas clínicamente sanas, sin antecedentes patológicos pulmonares.

Con estos resultados, se realizó una búsqueda bibliográfica que permitiese entender de mejor manera los hechos que se estaban observando. Se encontró que los efectos posteriores al cuadro agudo son los temas menos investigados, siendo escasa la bibliografía; por lo tanto, es importante estudiar la presencia de disfunción respiratoria a seis meses de seguimiento, en pacientes que fueron casos confirmados de NIAH1N1.

Enesesentido, elobjetivogeneraldelainvestigación fue determinar los factores de riesgo asociados a la presencia de alteraciones respiratorias clínicas, espirométricas y radiográficas, y las características de estos al sexto mes de seguimiento en sujetos con antecedente de infección confirmada por NIAH1N1, de julio de 2009 a febrero de 2010.

Los objetivos específicos fueron los siguientes:

$\Delta$ Describir los factores de riesgo y las características clínicas, radiográficas y espirométricas al sexto mes de seguimiento de los sujetos con antecedente de infección por la NIAH1N1.

- Determinar si la presencia de patología de riesgo asociada es un factor de riesgo para desarrollar alteraciones clínicas, radiográficas y espirométricas al sexto mes de seguimiento de los sujetos con antecedente de infección por la NIAH1N1.

- Determinar si la demora en la administración de terapia específica, es un factor de riesgo para desarrollar alteraciones clínicas, radiográficas y espirométricas. 
- Determinar si la presencia de insuficiencia respiratoria aguda grave (IRAG) es un factor de riesgo para desarrollar alteraciones clínicas, radiográficas y espirometricas.

\section{MATERIAL Y MÉTODOS}

Se desarrolló un estudio analítico de corte transversal, en 118 pacientes mayores de 14 años, a seis meses de seguimiento de haber tenido diagnóstico confirmado de NIAH1N1 por PCRtr (realizada por el Instituto Nacional de Salud), tratados durante julio-agosto 2009, quienes fueron atendidos por consulta externa o en emergencia por un cuadro respiratorio agudo, sin antecedente de afección pulmonar previa en 2009 , que habían acudido al Hospital Antonio Lorena y Hospital Regional del Cusco. El estudio se realizó en enero y febrero de 2010. La alteración respiratoria clínica fue evaluada a través de la saturación de oxígeno.

Los sujetos ingresaron al estudio de acuerdo a los criterios de inclusión y exclusión establecidos en el presente proyecto de investigación.

\section{Criterios de inclusión}

- Sujetos con diagnóstico de la NIAH1N1 con resultados positivo por PCRtr, evaluados en el Hospital Antonio Lorena del Cusco (por consultorio externo o servicio de emergencia).

- Sujetos mayores de 14 años.

- Sujetos sin afección broncopulmonar diferente a esta causa durante el 2009.

- Sujetos residentes y procedentes de la ciudad del Cusco durante el periodo de estudio.

- Sujetos que aceptaron ser parte del estudio, previo consentimiento informado.

\section{Criterios de exclusión}

$\Delta$ Antecedente de proceso no infeccioso que puede generar el cuadro previamente descrito.

$\triangle$ Antecedente de cuadro broncopulmonar durante 2009 de causa distinta a la estudiada.

- Sujetos con ocupaciones de riesgo para desarrollar enfermedades pulmonares crónicas.

- Sujetos con problemas de respuesta a órdenes.

- Sujetos que por algún motivo no tengan un llenado adecuado de sus historias clínicas o fichas de seguimiento, estén incompletas o que presenten deficiencias o inconsistencias.

El muestreo fue de tipo no probabilístico, por conveniencia.

Se identifico a 160 casos de NIAH1N1, confirmados por PCRtr, de los cuales se seleccionaron 118 sujetos que cumplían con los criterios de inclusión y exclusión.

Deestos 118 sujetosidentificadosquecorresponden a la muestra del estudio, 50 corresponden al Hospital Antonio Lorena y 68 al Hospital Regional.

El estudio se realizó mediante la revisión de las historias clínicas y fichas de seguimiento de los sujetos incluidos en el estudio; se utilizó una ficha de recolección de datos validada por seis médicos especialistas, que tenían relación con el manejo del daño en estudio. Los estudios de saturación de oxígeno y radiográficos se realizaron en cada hospital de estudio, en tanto que las pruebas de espirometría se realizaron en el Hospital Antonio Lorena.

La ficha de recolección de datos estaba divido en cuatro partes: a) datos de filiación, b) antecedentes patológicos de importancia, c) datos de la enfermedad y d) datos de los controles a los seis meses de seguimiento: ficha clínica, radiográfica y de espirometría

Todos los procedimientos se realizaron en cumplimiento de las normas internacionales de ética en la investigación y la normas de ética médica en la investigación, establecidas por el Colegio Médico del Perú. Se realizaron previo consentimiento informado, y respetando la privacidad y la confidencialidad de la información.

Con base en los datos recolectados en las fichas de investigación, se realizó una base de datos con el programa Excel MS. Se utilizó estadística descriptiva (número, porcentaje y media), para mostrar los resultados relacionados a las variables, los cuales se muestran en tablas y gráficos.

Para el análisis de la asociación de las variables cualitativas en estudio, se elaboró tablas de contingencia de $2 \times 2$, que fueron analizadas mediante el paquete estadístico Epi Info 3.5.1., para calcular la razón de prevalencia (RP), y así evaluar 


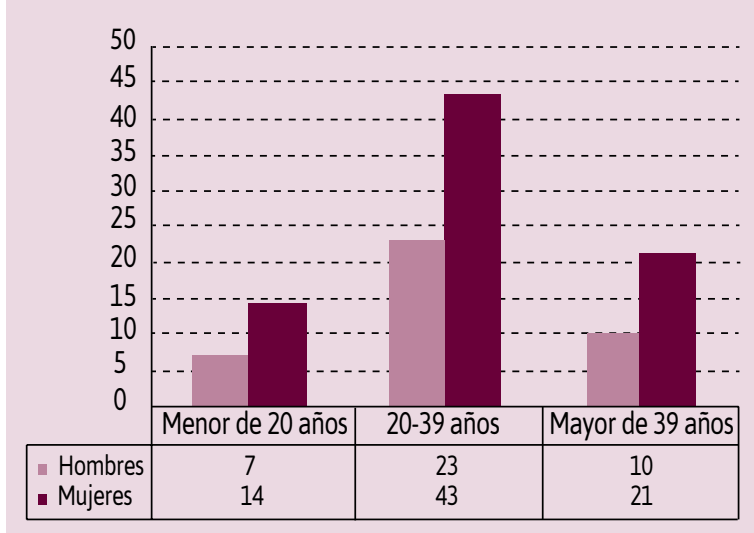

Figura 1. Sujetos con antecedente de infección por nueva influenza A (H1N1), según edad y sexo, Hospital Antonio Lorena y Hospital Regional del Cusco, 2009-2010.

la fuerza de la asociación de las variables; el ji cuadrado $(p<0,05)$ y los intervalos de confianza del $95 \%$ para evaluar la significancia estadística de la asociación.

\section{RESULTADOS}

Casi siete de cada diez sujetos presentó alguna alteración sea clínica, espirométrica o radiográfica, o una combinación de ellas, pero no existió diferencias en cuanto al género. Las alteraciones respiratorias espirométrica y clínica fueron las más frecuentes.

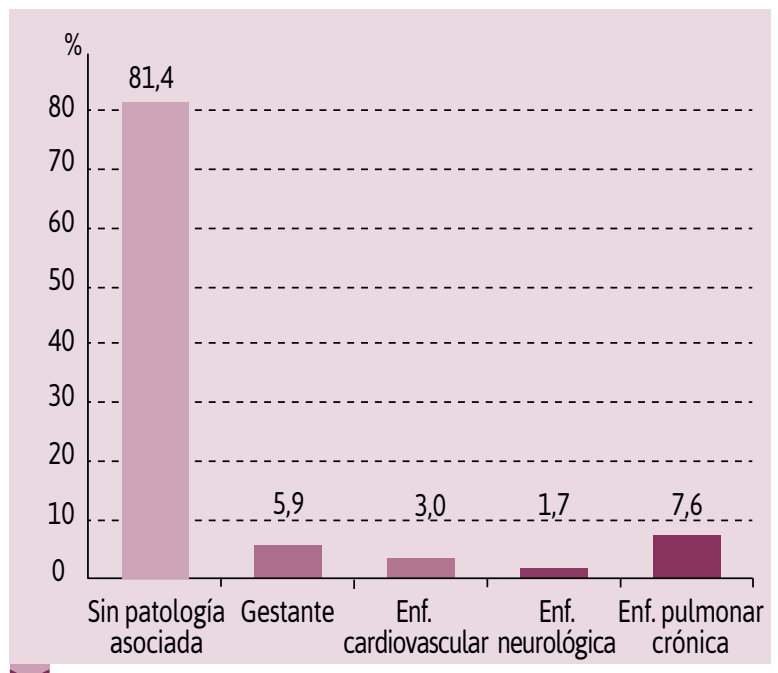

Figura 2. Tipos de patología de riesgo asociada en sujetos con antecedente de infección por nueva influenza A (H1N1), Hospital Antonio Lorena y Hospital Regional del Cusco, 2009-2010

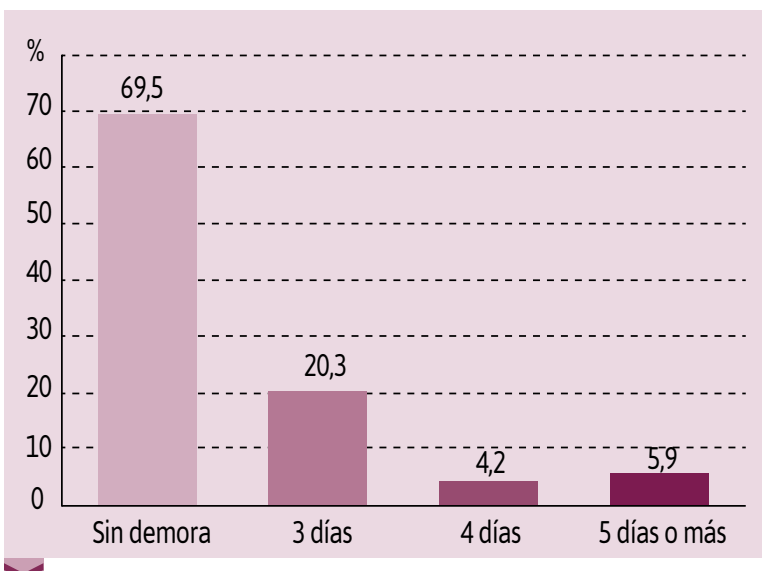

Figura 3. Demora en el inicio de tratamiento específico en sujetos con antecedente de infección por Nueva Influenza A (H1N1), Hospital Antonio Lorena y Hospital Regional del Cusco, 2009-2010

La IRAG se presentó en $49 \%$ de los sujetos con antecedente de infección por NIAH1N1.

La patología de riesgo asociada $(\mathrm{RP}=4,11$ [IC95\% = 2,49-6,78], $p=0,0000002)$, la demora en el inicio del tratamiento antiviral específico ( $\mathrm{RP}=4,56$ [IC95\% $=2,48-8,37], p=0,0000001$ ) y la presencia de IRAG (RP = 11,35 [IC95\% = 4,27-30,17], $p=0,0000000)$ son FRA a alteraciones clínicas; la patología de riesgo asociada ( $\mathrm{RP}=12,52 \quad$ [IC95\% $=2,7-58,08]$, $\mathrm{p}=0,00003)$, FRA a alteración radiográfica; la presencia de IRAG $(\mathrm{RP}=1,33$ [IC95\% = 1,02-1,74], $p=0,04)$, FRA a alteración espirométrica.

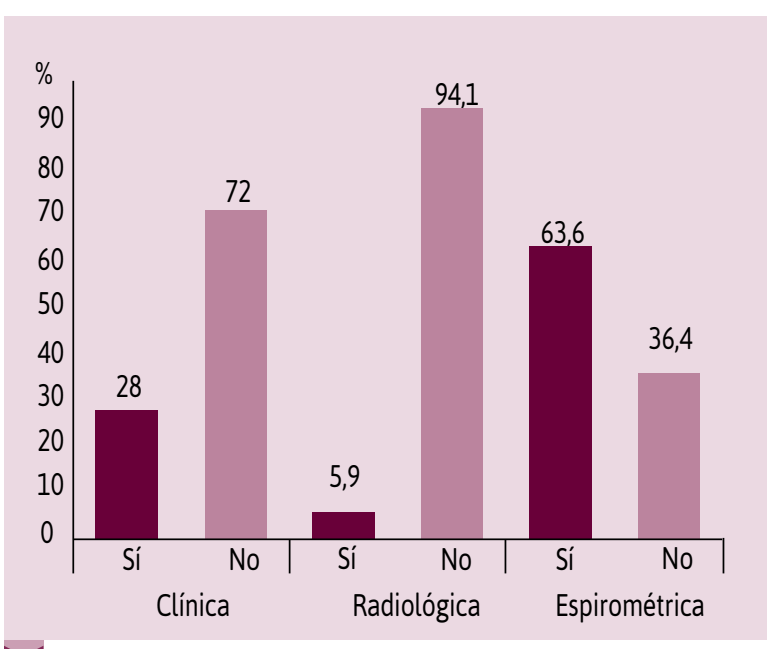

Figura 4. Tipo de alteración (clínica, radiográfica o espirométrica) a seis meses de seguimiento en sujetos con antecedente de infección por nueva influenza A (H1N1), Hospital Antonio Lorena y Hospital Regional del Cusco, 2009-2010 


\section{Anta Méálica Perwana}

Tabla 1. Presencia de alteración respiratoria clínica (medida por saturación de oxígeno) y su relación con factores de riesgo asociados, al sexto mes de seguimiento en sujetos con antecedente de infección por nueva influenza A (H1N1), Hospital Antonio Lorena y Hospital Regional del Cusco, 2009-2010.

\begin{tabular}{|c|c|c|c|c|c|c|c|c|c|c|}
\hline & & \multicolumn{4}{|c|}{ Alteración respiratoria clínica } & \multirow[b]{3}{*}{ RP } & \multirow[b]{3}{*}{$\chi^{2}$} & \multirow[b]{3}{*}{$P$} & \multicolumn{2}{|c|}{ IC95\% } \\
\hline \multicolumn{2}{|c|}{ Variable } & \multicolumn{2}{|c|}{ Sí } & \multicolumn{2}{|c|}{ No } & & & & \multirow{2}{*}{\multicolumn{2}{|c|}{ Inferior Superio }} \\
\hline & & $\mathrm{N}$ & $\%$ & $\mathrm{~N}$ & $\%$ & & & & & \\
\hline \multirow[t]{2}{*}{$\triangle$ Sexo } & Femenino & 24 & 30,8 & 54 & 69,2 & 1,03 & 0,01 & 0,93 & 0,57 & 1,83 \\
\hline & Masculino & 12 & 30,0 & 28 & 70,0 & & & & & \\
\hline \multirow[t]{2}{*}{$\triangle$ Edad de riesgo } & Sí & 15 & 22,7 & 51 & 77,3 & 0,7 & 1,46 & 0,22 & 0,38 & 1,26 \\
\hline & No & 17 & 32,7 & 35 & 67,3 & & & & & \\
\hline \multirow{2}{*}{$\begin{array}{l}\text { Patología de } \\
\text { riesgo asociada }\end{array}$} & Sí & 16 & 72,7 & 6 & 27,3 & 4,11 & 26,89 & 0,0000002 & 2,49 & 6,78 \\
\hline & No & 17 & 17,7 & 79 & 82,3 & & & & & \\
\hline \multirow{2}{*}{$\begin{array}{l}\text { Demora en inicio } \\
\text { de tratamiento } \\
\text { específico }\end{array}$} & Sí & 22 & 61,1 & 14 & 38,9 & 4,56 & 28,25 & 0,0000001 & 2,48 & 8,37 \\
\hline & No & 11 & 13,4 & 71 & 86,6 & & & & & \\
\hline \multirow[t]{2}{*}{$\triangle$ Presencia de IRAG } & Sí & 29 & 63,0 & 17 & 37,0 & 11,35 & 46,05 & 0,0000000 & 4,27 & 30,17 \\
\hline & No & 4 & 5,6 & 68 & 94,4 & & & & & \\
\hline
\end{tabular}

IRAG: infección respiratoria aguda grave

\section{DISCUSIÓN}

El presente estudio contó con la participación de 118 sujetos con antecedente de infección por la
NIAH1N1, cuya muestra es superior a las revisiones publicadas sobre el tema a la fecha. ${ }^{20,21}$ Se evaluó a sujetos hospitalizados y de manejo ambulatorio, importante diferencia, ya que los estudios

Tabla 2. Presencia de alteración radiográfica y su relación con factores de riesgo asociados, al sexto mes de seguimiento en sujetos con antecedente de infección por nueva influenza A(H1N1), Hospital Antonio Lorena y Hospital Regional del Cusco, 2009-2010.

\begin{tabular}{|c|c|c|c|c|c|c|c|c|c|c|c|}
\hline \multirow{3}{*}{\multicolumn{2}{|c|}{ Variable }} & & \multicolumn{4}{|c|}{ Alteración radiográfica } & \multirow{3}{*}{$\mathrm{RP}$} & \multirow{3}{*}{$\chi^{2}$} & \multirow{3}{*}{$\mathrm{P}$} & \multirow{2}{*}{\multicolumn{2}{|c|}{ IC 95\% }} \\
\hline & & & \multicolumn{2}{|c|}{ Sí } & \multicolumn{2}{|c|}{ No } & & & & & \\
\hline & & & $\mathrm{N}$ & $\%$ & $\mathrm{~N}$ & $\%$ & & & & Inferior & Superior \\
\hline \multirow{2}{*}{\multicolumn{2}{|c|}{$\triangle$ Sexo }} & Femenino & 2 & 3 & 76 & 97 & 0,21 & 4,68 & 0,03 & 0,04 & 1,01 \\
\hline & & Masculino & 5 & 13 & 35 & 88 & & & & & \\
\hline \multirow[t]{2}{*}{$\Delta$} & \multirow[t]{2}{*}{ Edad de riesgo } & Sí & 0 & 0 & 66 & 100 & \multirow{2}{*}{$\begin{array}{l}\text { No calculado } \\
\text { (NC) }\end{array}$} & \multirow[t]{2}{*}{10,68} & \multirow[t]{2}{*}{0,001} & \multirow[t]{2}{*}{ NC } & \multirow[t]{2}{*}{ NC } \\
\hline & & No & 8 & 15 & 45 & 85 & & & & & \\
\hline \multirow{2}{*}{$\Delta$} & \multirow{2}{*}{$\begin{array}{l}\text { Patología de } \\
\text { riesgo asociada }\end{array}$} & Sí & 6 & 26 & 17 & 74 & \multirow[t]{2}{*}{12,52} & \multirow[t]{2}{*}{17,05} & \multirow[t]{2}{*}{0,00003} & \multirow[t]{2}{*}{2,7} & \multirow[t]{2}{*}{58,08} \\
\hline & & No & 2 & 2 & 94 & 98 & & & & & \\
\hline \multirow{2}{*}{$\Delta$} & \multirow{2}{*}{$\begin{array}{l}\text { Demora en inicio de } \\
\text { tratamiento específico }\end{array}$} & Sí & 7 & 19 & 29 & 81 & \multirow[t]{2}{*}{ NC } & \multirow[t]{2}{*}{16,95} & \multirow[t]{2}{*}{0,00003} & \multirow[t]{2}{*}{ NC } & \multirow[t]{2}{*}{ NC } \\
\hline & & No & 0 & 0 & 82 & 100 & & & & & \\
\hline \multirow{2}{*}{$\Delta$} & \multirow{2}{*}{$\begin{array}{l}\text { Presencia de insuficiencia } \\
\text { respiratoria aguda grave }\end{array}$} & Sí & 7 & 15 & 39 & 85 & \multirow[t]{2}{*}{ NC } & \multirow[t]{2}{*}{11,65} & \multirow[t]{2}{*}{0,0006} & \multirow[t]{2}{*}{$\mathrm{NC}$} & \multirow[t]{2}{*}{ NC } \\
\hline & & No & 0 & 0 & 72 & 100 & & & & & \\
\hline
\end{tabular}


considerados como antecedentes, evaluaron solo a sujetos hospitalizados,. Esta característica es un hecho que explica los resultados propios del estudio.

Las limitaciones del estudio estuvieron relacionadas a lo siguiente:

- Los escasos estudios relacionados al tema de secuelas, que permitan establecer comparaciones.

- La no existencia de un protocolo de seguimiento de los pacientes que hayan tenido un diagnóstico de NIAH1N1.

- Limitación de recursos económicos para realizar estudios complementarios como pletismografía, tomografía axial computarizada contrastadas que podrían aportar datos de mayor precisión.

$\triangle$ El contar con registros hospitalarios, historias clínicas o fichas de seguimienton incompletas o que presenten deficiencias o inconsistencias.

Sin embargo, hay que destacar que no existían referencias sobre este tipo de estudio en la bibliografía internacional. Se consideran de importancia los resultados encontrados, y seguro servirán de base para estudios futuros más amplios y de diferente naturaleza.

Las características demográficas vistas en la Figura 1 fueron diferentes a las descritas por la literatura. El presente estudio encontró diferencia en la frecuencia según sexo sexo $(66,1 \%$ de población femenina; relación mujer/varón de 1,95), en tanto que Vaquéz2 reportó que la relación entre varón y mujer fue de 1 . En la población de mujeres, se encontró que 4 de ellas, se encontraban en estado de gestación.

En cuanto a la edad, se pudo apreciar el predominio del grupo etario de 20 a 39 años $(55,9 \%)$ y una menor frecuencia en los grupos a partir de 50 años. Este hallazgo fue muy semejante a lo reportado por Navarro, ${ }^{20}$ que encontró como edad media de afectación en mayores de 35 años. Para Vaqué, 22 $78,7 \%$ de los casos acumulados confirmados tenían menos de 30 años. Asimismo, Quirita et al. encontraron en su serie, que la mayor frecuencia de casos se dio en las edades comprendidas entre los 20 y 39 años.
Como se puede apreciar nuestros hallazgos y los descritos coinciden en que el grupo etario más afectado corresponde a los menores de 40 años, hecho que difiere con lo conocido para el caso de la influenza estacional, que toma a los extremos de vida la vida como casos de mayor frecuencia. Es más, la OMS reconoce que la edad de riesgo es la comprendida entre los 20 y 39 años.

En cuanto a la presencia de patología de riesgo asociada (ver Figura 2), se aprecia que $81,4 \%$ de los sujetos en estudio no la tenían y el restante $18,6 \%$ presentaron alguna patología de riesgo asociada, lo que difiere con lo reportado por Navarro, ${ }^{20}$ quien encontró patología de riesgo asociada en $73 \%$ de su muestra en población estadounidense, y $48 \%$ en Chile; a su vez, Vaqué22 observó que $41 \%$ de sus casos presentaron patología de riesgo asociada. Esta diferencia marcada entre los estudios previos, y el presente estudio puede atribuirse a la población hospitalizada en la que se realizaron dichos estudios, a diferencia del presente estudio que evaluó tanto la población hospitalizada como la ambulatoria.

De los 22 sujetos ( $18,6 \%$ del total) en estudio que tuvieron alguna patología de riesgo asociada, 40,9\% tenían enfermedad pulmonar crónica; $31,8 \%$, gestación; 18,2\%, enfermedad cardiovascular; frecuencias que son muy semejantes a lo descrito por la literatura internacional. . $^{2,22}$

En cuanto a la demora en el tratamiento específico (Figura 3), se pudo ver que $69,5 \%$ de los sujetos en estudio no tuvieron demora en el inicio del tratamiento antiviral específico; en tanto que $20,3 \%$ recibieron el tratamiento al tercer día; $4,2 \%$, al cuarto día; $5,9 \%$, al quinto día o más después del inicio de síntomas. En lo encontrado por Vaqué, 22 se calculó la letalidad ajustada con el retraso de diagnóstico, y, por tanto, de tratamiento. Se encontró 0,43\% para México y $0,68 \%$ para los casos de EE UU. Coincidimos con Vaqué al manifestar que resulta difícil comparar dicho resultado ya que los países no captan ni confirman los casos moderados a leves. Grajeda et al.,. ${ }^{23}$ en su estudio, encontraron que $55 \%$ de los pacientes en estudio, recibieron el tratamiento antiviral específico hasta el tercer día después de iniciado los síntomas. 
Respecto a la presencia IRAG (Figura 4), que fue indicación de hospitalización, en los pacientes en estudio que tuvieron un cuadro agudo de NIAH1N1, encontramos que $49 \%$ de ellos tuvieron un cuadro de IRAG. Esta frecuencia es mayor a la descrita por Navarro, ${ }^{20}$ quien reportó de $2 \%$ a $7 \%$ del total de sujetos con IRAG; a su vez, Vaquéz reportó 6\%; la diferencia marcada podría deberse a algunos factores como criterios heterogéneos de ingreso hospitalario, capacidad de camas hospitalarias, categoría de hospital, si el hospital es de referencia o no, entre otros, por lo cual los datos deben interpretarse con precaución.

Respecto a las alteraciones respiratoria clínica, radiográfica y espirométrica observadas a seis meses de seguimiento de los sujetos en estudio (Figura 5), se aprecia que $28 \%$ presentaron alteración respiratoria clínica medida por la saturación de oxígeno igual o menor de $89 \% ; 5,9 \%$, alguna alteración radiológica; $63,6 \%$, algún tipo de alteración espirométrica. Eso significa que, de todos los sujetos que hayan padecido un cuadro agudo de NIAH1N1 tendrían una alta probabilidad de presentar algún tipo de alteración clínica o radiológica o espirométrica al sexto mes, por lo que debería hacerse el seguimiento a todo sujeto infectado, según la normatividad clínica respectiva.

A la presencia de alteración respiratoria clínica (medida por saturación de oxígeno) y su relación con factores de riesgo asociados al sexto mes de seguimiento en sujetos con antecedente de infección por NIAH1N1 (Tabla 1), se aprecia que los sujetos en estudio con antecedente de infección por NIAH1N1 a seis meses de seguimiento, $28 \%$ de ellos presentaron alteración respiratoria clínica, hecho que no se ha podido contrastar, debido que no se pudo encontrar reportes de investigaciones previas, los que muestran la necesidad de contar con un protocolo de seguimiento de todo sujeto que haya tenido infección por NIAH1N1.

El estudio mostró que no existe asociación entre la presencia de alteración respiratoria clínica con el sexo (razón de prevalencia $=1,03 ; p=0,93$; $[$ IC95\% $=0,57-1,83])$ y la edad de riesgo $(\mathrm{RP}=0,7$; $\mathrm{p}=0,22 ;[$ [C95\% = 0,38-1,26]).

El estudio mostró que, tener una patología de riesgo en los pacientes con NIAH1N1 es un factor de riesgo para que a los seis meses de seguimiento, una persona presente una alteración respiratoria clínica, con una razón de prevalencia (RP) de 4,11, lo que significa que las personas con patología de riesgo y de haber padecido NIAH1N1 tienen 4,1 veces más riesgo de desarrollar una alteración clínica a los seis meses que las personas que no tienen una patología de riesgo asociada, siendo esta diferencia estadísticamente significativa ( $p=0,0000002 ;$ [IC95\% = 2,49-6,78]).

Las personas que tuvieron demora en el inicio del tratamiento antiviral específico tuvieron 4,5 veces más riesgo $(\mathrm{RP}=4,5)$ de tener una alteración respiratoria clínica respecto a las personas que no tuvieron demora en el inicio del este tratamiento. Esta diferencia es estadísticamente significativa ( $p=0,000001 ;[$ IC95\% = 2,48-8,37]); por lo tanto, la demora en el inicio del tratamiento es un factor de riesgo a considerar para la presencia de alteración respiratoria clínica a los seis meses de seguimiento; estos resultados pueden explicarse con lo descrito por Navarro ${ }^{20}$ y Grajeda ${ }^{23}$ cuando evalúan los factores de riesgo asociados a mayor mortalidad.

La presencia de IRAG en la fase aguda de los pacientes con NIAH1N1 es un factor de riesgo a los seis meses de seguimiento de estos pacientes. $(R P=11,35 ; p=0,0000000 ;[$ IC 95\% = 4,27-30,17]).

Con respecto a la presencia de alteración radiográfica y su relación con factores de riesgo asociados al sexto mes de seguimiento en sujetos con antecedente de infección por NIAH1N1 (Tabla 2), 5,9\% presentaron alteración radiográfica, hecho que no se ha podido comparar, debido a que no se pudo encontrar reportes de investigaciones previos.

El estudio mostró que no existe asociación entre la presencia de alteración radiográfica torácica con el sexo (RP = 0,21; $p=0,03$; [IC95\% = 0,04-1,01]), con la edad de riesgo ( $R P=$ no calculado; $p=0,001$; [IC95\% = NC-NC]), con la demora en el inicio del tratamiento $(\mathrm{RP}=\mathrm{NC} ; \mathrm{p}=0,00003 ; \quad[\mathrm{IC} 95 \%=\mathrm{NC}$ $\mathrm{NC}])$, ni con la presencia de IRAG (RP $=\mathrm{NC}$; $p=0,0006 ;[$ [IC95\% = NC-NC]).

Tener una patología de riesgo asociada en los pacientes con NIAH1N1 es un factor de riesgo para que a los seis meses de seguimiento una persona presente una alteración radiográfica torácica, con 
una RP de 12,52, lo que significa que las personas con patología de riesgo y haber padecido NIAH1N1 tienen 12,5 veces más riesgo de desarrollar una alteración radiográfica a los seis meses que las personas que no tienen patología de riesgo asociada, siendo esta diferencia estadísticamente significativa ( $p=0,001 ;[$ [IC95\% = 2,7-58.1]).

La alteración radiográfica se presentó en un bajo porcentaje de los sujetos de estudio (5,9\%), lo que coincide con lo descrito por Pracchi,, ${ }^{21}$ que en 66 sujetos con infección por NIAH1N1 encontró normalidad en la placa radiográfica, pero que en la tomografía computarizada contrastada de tórax 10 sujetos tuvieron embolismo pulmonar. Coincidimos en manifestar que la radiografía de tórax no sería un buen método diagnóstico para estos sujetos; la tomografía es el mejor método para el seguimiento, lo que podría explicar la escasa frecuencia de presentación para nuestro estudio.

Es necesario mencionar que las alteraciones radiográficas se presentaron en los sujetos que contaban con antecedente de enfermedad cardiovascular y de enfermedad crónica pulmonar mostrando infiltrado segmentario lobar que compromete a un solo cuadrante; por lo tanto, consideramos que no es posible evaluar factores de riesgo para la presencia de alteraciones radiográficas ni concluir al respecto. Además, se sugiere no considerar a la radiografía de tórax un método diagnóstico para valorar el sistema respiratorio dentro del protocolo de seguimiento.

En relación a la presencia de alteración espirométrica y su relación con factores de riesgo asociados, al sexto mes de seguimiento en sujetos con antecedente de infección por NIAH1N1 (Tabla 3), se aprecia que $63,6 \%$ presentaron alteración espirométrica, resultado que no se ha podido contrastar, por no haber podido encontrar referencias de estudios previos.

El estudio mostró que no existe asociación entre la presencia de alteración espirométrica con el sexo $(\mathrm{RP}=0,93 ; p=0,59 ;[\mathrm{IC} 95 \%=0,71-1,21])$, con la edad de riesgo ( $R P=0,75 ; p=0,03$; [IC95\% $=0,57-0,98]$ ), con la patología de riesgo asociada $(R P=1,28$; $\mathrm{p}=0,13$; [IC95\% $=0,97-1,69]$ ), ni con la demora en el inicio de tratamiento antiviral especifico $(R P=1,21 ; p=0,195 ;[$ IC95\% = 0,92-1,58]).
Haber tenido un cuadro de IRAG en los pacientes con NIAH1N1 es un factor de riesgo para que a los seis meses de seguimiento, una persona presente una alteración espirométrica, con una $\mathrm{RP}$ de 1,33 , lo que significa que las personas con patología de riesgo y haber padecido la NIAH1N1 tienen 1,33 veces más riesgo de desarrollar una alteración espirométrica a los seis meses que las personas que no tienen una patología de riesgo asociada, siendo esta diferencia estadísticamente significativa ( $p=0,04 ;[$ IC95\% = 1,02-1,74]).

Dado que no se encontró relación entre el sexo, la edad de riesgo, la patología de riesgo asociada y la demora en inicio de tratamiento antiviral específico con la presencia de alteraciones espirométricas Esto significa que cualquier sujeto con infección por este virus puede desarrollar alteraciones de este tipo independientemente de estos factores, lo que coincide con lo reportado por Fariña. ${ }^{18}$

Referente a la presencia del tipo de alteración espirométrica y su relación con factores de riesgo asociados, al sexto mes de seguimiento en sujetos con antecedente de infección por NIAH1N1 (Cuadro 4), se puede apreciar que la alteración espirométrica del tipo obstructivo es la más frecuente y se aprecia que la relación es mayor respecto a la edad de riesgo, patología de riesgo asociada, demora de inicio de tratamiento antiviral especifico y presencia de IRAG, esta relación es estadísticamente significativa.

Dado que el trabajo muestra un importante frecuencia de alteración espirométrica del tipo obstructivo, con frecuencias semejantes entre la población hospitalizada y los casos ambulatorios, este hallazgo podría asociarse con la presencia de patología pulmonar crónica de los sujetos que pertenecen a este grupo, en tanto que la presencia de patrón mixto espirométrico se presentó en un porcentaje semejante al restrictivo, lo que fue mayor en la población atendida ambulatoriamente. En los pacientes con IRAG, hubo un mayor patrón restrictivo y obstructivo, con frecuencias semejantes.

En relación a los tipos de alteración espirométrica, se observó que existe fuerte asociación entre la presencia de patología de riesgo asociado, la demora en el tratamiento y la presencia de IRAG para el patrón restrictivo y obstructivo, hallazgo 
semejante a lo descrito por Fariña (18), el cual indicó la presencia del patrón restrictivo de severidad variable en los sujetos hospitalizados en terapia intensiva. Es por ello que sugerimos considerar dentro del protocolo de seguimiento a la prueba espirométrica y el realizar en aquellos que resulten alterados tres pruebas que confirmen la presencia del patrón espirométrico restrictivo.

\section{CONCLUSIONES}

$\triangle$ La nueva influenza A (H1N1) [NIAH1N1] produce alteraciones clínicas, espirométricas y radiográficas a seis meses de presentarse el cuadro agudo en un importante numero de casos.

$\Delta$ En los sujetos con infección por la NIAH1N1 la mayoría presentaron algún tipo de alteración, sin que exista mayor variación en cuanto al sexo, siendo la alteración espirométrica la más frecuente, predominando el patrón obstructivo, seguida de la alteración clínica y finalmente la alteración radiográfica.

- Existen factores de riesgo como patología de riesgo asociada, demora en el inicio del tratamiento antiviral específico e insuficiencia respiratoria aguda grave (IRAG) que requieren ser considerados, para dar tratamiento apropiado y realizar el seguimiento respectivo, y prevenir estas alteraciones.

- La patología de riesgo asociada, la demora en el inicio del tratamiento antiviral específico y la presencia de IRAG en pacientes que tuvieron NIAH1N1 a seis meses de seguimiento, son factores de riesgo asociados a la presencia de alteración respiratoria clínica.

- La patología de riesgo asociada es un factor de riesgo para la presencia de alteración radiográfica, y la presencia de IRAG es un factor de riesgo para la presencia de alteración espirométrica en pacientes que tuvieron NIAH1N1 a seis meses de seguimiento; así como que la presencia de patología de riesgo asociada esta relacionada más a alteración espirométrica de patrón obstructivo.

- No se puede concluir sobre las características de las alteraciones radiográficas.

\section{SUGERENCIAS}

- Elaborar un protocolo de seguimiento a los sujetos afectados por el virus de la NIAH1N1 teniendo como grupos de riesgo a los sujetos con patología de riesgo asociada, principalmente aquellos que presenten antecedente de enfermedad pulmonar crónica, enfermedad cardiovascular y el estado de gestación. Además de considerar a los sujetos que desarrollen IRAG.

- No considerar a la radiografía de tórax como método diagnóstico de seguimiento, ya que no mostró mayor alcance para el reconocimiento de alteraciones radiográficas en sujetos con alteraciones clínicas y espirométricas establecidas.

- Que la evaluación por espirometría consista en tres mediciones a fin de corroborar las alteraciones en el patrón.

- Concientizar a la población sobre los riesgos existentes posteriores al cuadro agudo, a fin de tener un mejor control de las secuelas.

- Continuar el estudio con un diseño de cohorte prospectivo que permita identificar los factores asociados a la presencia de las alteraciones ya descritas. 


\section{REFERENCIAS BIBLIOGRÁFICAS}

I. Organización Panamericana de la Salud. Consulta de expertos de OPS/OMS: recomendaciones provisionales para el manejo clínico de la Influenza pandémica (HINI). Organización Panamericana de la Salud Washington, USA. reemplaza la del 26 de mayo de 2009. Sao Pablo: OPS; 2009. [Página en Internet]. Accesado 01/04/2014. Disponible en http://www.scielo.org.pe/ pdf/amp/v30n4/a08v30n4.pdf

2. Bresee J. "La Gripe A (HINI) 2009 ("gripe porcina") y Usted". [National Center for Immunization and Respiratory Diseases, Influenza Division (NCIRD/ID)]. USA. Centros para el Control y la Prevención de Enfermedades. 2009. Accesado el 0I/01/2014 Disponible en : http://www.cdc.gov/CDCTV/SwineFlu

3. Camarena S.Alarma en México y EE UU por una rara influenza porcina. [Periodico en Internet El País.com]. 25 abril 2009. Accesado el 0I/0I/I4. Disponible en URL: http://sociedad.elpais. com/sociedad/2009/04/25/actualidad/I2406/040I_8502/5.html

4. OMS. Preparing for the second wave: lessons from current outbreaks. [sede Web]. Geneva. 2009. Accesada el 0I/0I/I4. Disponible en http://www.who.int/csr/disease/swineflu/notes/ hInl_second_wave_20090828/en/

5. Chan M. El nivel de alerta de pandemia de gripe porcina pasa a la fase 4 Declaración de la Directora General de la OMS sobre Gripe porcina, 27 de abril de 2009 Disponible en URL: http://www.who.int/mediacentre/news/statements/2009/h In I_ pandemic_phase6_200906II/es/

6. Chotani RA. Influenza $\mathrm{A}(\mathrm{HINI})$ (gripe porcina) pandémica. [Presentación Internet]. En 'Justo en tiempo'. EE UU. 2009. Accesado el $0 \mathrm{I} / 0 \mathrm{I} / \mathrm{I} 4$. Disponible en www.bibalex.org/ supercourse/supercourseppt/340 I I-3500 I/3462I.ppt

7. Cunha BA. Influenza: historical aspects of epidemics and pandemics. Infect Dis Clin North Am. 2004; 18(I): 14I-55.

8. Dirección Regional de Salud Cusco, Declaratoria de Alerta Epidemiológica. Oficio circular N. 04372009 - GR Cusco DRSC. DEUS-EPI 27 de abril de 2009.

9. Directiva Sanitaria N..$^{\circ}$ 027-Minsa/DGSP.V.0I. Directiva Sanitaria para la Atención, diagnóstico y tratamiento de influenza en los establecimientos de salud a nivel nacional en la etapa de mitigación de la pandemia influenza por virus $A(\mathrm{HINI})$.

10. Lipsitch M, Riley S, Cauchemez S, Ghani AC, Ferguson NM. Managing and reducing uncertainty in an emerging influenza pandemic. N Engl J Med. 2009;36 I (2): I 2I- I I5.

II. Ong CWM, Ho KY, Hsu LY, Lim AYT, Fisher DA, Tambyah PA. Reacting to the emergence of swine-origin influenza A HINI. Lancet Infect Dis. 2009;9(7):397-8.

12. Laboratorio suizo produce primer lote de vacunas contra Influenza humana. Emol.Mundo. [diario en internert] 12 de junio de 2009,Noticias.Accesado el 0 I/0 I/ I4.Disponible en URL:http:// www.emol.com/noticias/internacional/2009/06//2/362464/ laboratorio-suizo-produce-primer-lote-de-vacunas-contrainfluenza-humana.html

13. Clemente Garulo D, Domínguez Ortega G. Pruebas para la detección rápida del virus de la gripe (v.2/2008). Guía_ABE.
Infecciones en Pediatría. Guía rápida para la selección del tratamiento antimicrobiano empírico [en línea] [actualizado el 29/I2/2008; accesado el 0I/0I//4]. Disponible en: http:// www.guia-abe.es/files/pdf/gripe_pruebas_deteccion_rapida_ v2_2008_anexo.pdf

14. Gómez $\bar{B}$. La pandemia de la gripe $\mathrm{A}(\mathrm{HINI})$ : ¿qué debemos saber? Situación mundial y en el Perú de la influenza A (HINI). [Presentación internet]. Perú. 2009. Accesado el 0I/0I//4. Disponible en:http://www.upch.edu.pe/tropicales/capacita/2009/ influenza/presentaciones/gomez.pdf

15. Gotuzzo E. ¿Qué lecciones tenemos en la pandemia actual y qué hacer? [videocinta]. Perú. 2009.Accesado el 0I/0I/I4. Disponible en: https://www.youtube.com/watch?v=-DHKyWNgqh8

16. Martino O. La situación de la epidemia de influenza $A(H I N I)$. Intramed. [sede Web]. Argentina. 2009. Accesado el 0I/0I//4. Disponible en: http://www.intramed.net/contenidover.asp? contenidoID $=60893$

17. Dispersión de los microorganismos: Reservorios y fuentes de infección. El contagio y su prevención. Vías y modos de transmisión microorganismos. En: Microbiología Clínica, Grupo de Investigación de Genética y Microbiología, Curso 2004-2005. [base de datos en Internet]. Universidad de Navarra. España. 2004-2005. Accesado el 0l/0I/I4. Disponible en: http://www. unavarra.es/genmic/microclinica/tema06.pdf

18. Fariña O, Pistillon N, Dorfman B, et al. Comentarios sobre la infección por nueva influenza $A$ (HINI) desde la perspectiva de la Terapia intensiva de adultos". Revista Científica Hospital El Cruce. 2009; I (5): [revista en Internet sin paginación] Accesado el 0I/0I//4. Disponible en: http://www.hospitalelcruce.org/ revis $5 / \mathrm{r} 5 \mathrm{~h} / \mathrm{nl}$.pdf

19. Organización Mundial de la Salud. Nota informativa N. ${ }^{\circ} 13$. [Sede web en Internet]. Cuadro clínico de los casos graves de gripe pandémica (HINI). Ginebra. 2009. Accesado el 0I/0I//4. Disponible en: http://www.who.int/csr/disease/swineflu/notes/ hInI_clinical_features_20091016/es/

20. Navarro-Marí JM, Mayoral JM, Pérez-Ruiz M, Rodríguez-Baño J, Carratalá J, Gallardo-García V. Infección en humanos por virus de la gripe $\mathrm{A}(\mathrm{HINI})$ : revisión al 30 de octubre de 2009 . Enferm Infecc Microbiol Clin. 2010;28:446-52.

21. University of Michigan Health System. Those with severe HINI at risk for pulmonary emboli, researchers find. [base de datos en Internet]. ScienceDaily. USA. 2009. Accesado el 01/0I/I4. Disponible en: www.sciencedaily.com/ releases/2009/10/0910141 I 1549.htm

22. Vaque Rafart J, Gil Cuesta J, Brotons Agullo M. Principales características de la pandemia por el nuevo virus nueva influenza A (HINI). Med Clin (Brac). 2009;133(I3):5I3-52I. [Articulo en Internet de datos en Internet].Accesado el 0I/0I//4. Disponible en: http://rafalafena.files.wordpress.com/2009//0/h I n -caracte risticasde-la-pandemia-por-el-nuevo-virus-influenza-a-h In l-mc-2009.pdf

23. Grajeda P. Factores de riesgo asociado a mortalidad por nueva influenza $A(\mathrm{HINI})$ en la región Cusco-Peru, 2009-20I0. En: Cuaderno de Resúmenes de la VII Conferencia Tephinet para Las Americas. Honduras. 20I I.

\section{Correspondencia}

Pablo Grajeda-Ancca pgrajedaa@hotmail.com

Fecha de recepción: 9 de mayo de 2014 Fecha de aceptación: 23 de junio de 2014

\section{Conflictos de interés}

Los autores declaran no tener conflictos de interés durante el planteamiento, ejecución de la investigación y la elaboración del artículo para su publicación. 